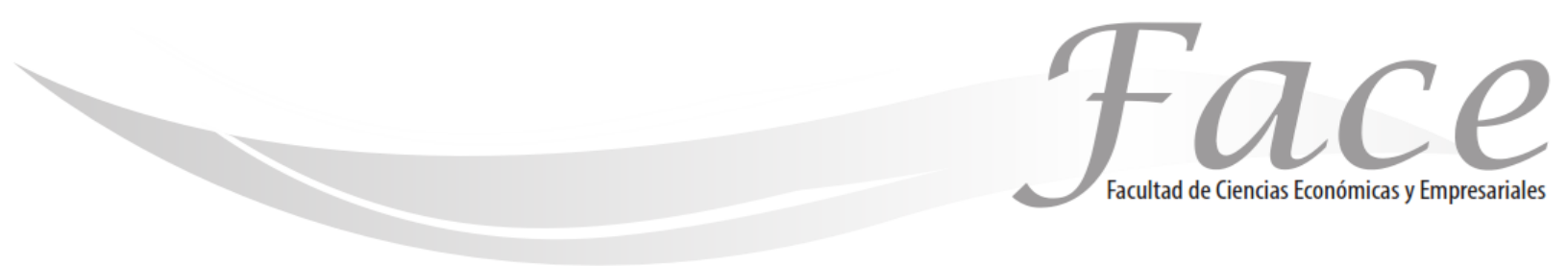

ISSN Impreso: 1794-9920

ISSN Electrónico: 2500-9338

Volumen $17-\mathrm{N}^{\circ} 2$

Año 2017

Págs.130-141

\title{
POLITICAS DE GESTION DE TALENTO HUMANO PARA EL DESARROLLO DE COMPETENCIAS GERENCIALES EN EMPRESAS METALMECANICA.
}

\author{
García Guiliany Jesús Enrique ** \\ Enlace ORCID: http://orcid.org/ 0000-0003-3777-3667 \\ Duran, Sonia Ethel ${ }^{* * *}$ \\ Enlace ORCID: http://orcid.org/0000-0002-0876-7850 \\ Prieto Pulido Ronald ${ }^{* * * *}$ \\ Enlace ORCID: http://orcid.org/0000-0003-3901-4250.
}

Fecha de Recepción: 25 noviembre 2016

Fecha de Aprobación: 6 febrero 2017

\section{Resumen:}

El estudio se orientó en analizar las políticas de gestión de talento humano en el desarrollo de las competencias gerenciales del personal supervisorio en empresas metalmecánica. La teoría se sustentó en Alles (2011), Chiavenato (2009), Hay Group (2012), entre otros. La investigación fue descriptiva, de campo, diseño no experimental, transversal, un cuestionario dirigido a 35 supervisores, con 30 items, con escala likert. La confiabilidad de 0.84 . Se obtuvo como resultado que en las empresas existen planes de captación de candidatos, y que estas políticas incluyen fuentes de reclutamiento, dentro y fuera de la organización, utilizando bases de datos, además, cuentan con políticas o programas de retención que permiten mantener a los buenos empleados; se establecen planes de incentivos salariales, la promoción del crecimiento profesional del supervisor. En cuanto a las competencias, se evidencia el trabajo en equipo, la cooperación y el liderazgo. Se concluyó que, en las organizaciones objeto de estudio están orientados a ejecutar los procesos de gestión de talento humano en forma coordinada, en virtud de promover el desarrollo de competencias.

Palabras Claves: Gestión; talento humano; políticas; competencias; gerencia.

** Doctor en ciencias gerenciales, Correo electrónico: jesus.garcia@unisimonbolivar.edu.co.

* Doctor en ciencias gerenciales, Correo electrónico: Soniaduran547@hotmail.com

*Doctor en ciencias gerenciales, Correo electrónico: rprieto1@unisimonbolivar.edu.co. 


\title{
HUMAN TALENT MANAGEMENT POLICIES FOR THE DEVELOPMENT OF MANAGEMENT COMPETENCES IN METALMECANICA COMPANIES.
}

\begin{abstract}
The study focused on the analysis of human talent management policies in the development of managerial competencies of supervisory personnel in metalworking companies. The theory was based on Alles (2011), Chiavenato (2009), Hay Group (2012), among others. The research was descriptive, field, non experimental design, transversal, a questionnaire addressed to 35 supervisors, with 30 items, with likert scale. The reliability of 0.84 . As a result, there are recruitment plans in the companies, and these policies include sources of recruitment, inside and outside the organization, using databases, in addition to having policies or retention programs that allow Good employees; salary incentive plans are established, promoting the professional growth of the supervisor. In terms of skills, teamwork, cooperation and leadership are evident. It was concluded that, in the organizations under study, they are oriented to execute human talent management processes in a coordinated way, by virtue of promoting the development of competencies.

Key words: Management; human talent; policies; competencies; management.
\end{abstract}

\section{POLÍTICAS DE GESTÃO DE TALENTOS HUMANOS PARA O DESENVOLVIMENTO DE COMPETÊNCIAS DE GESTÃO EM EMPRESAS DE METALMECANICA.}

\begin{abstract}
Resumo
O estudo centrou-se na análise de políticas de gestão de talento humano no desenvolvimento de competências gerenciais de pessoal de supervisão em empresas metalúrgicas. A teoria foi baseada em Alles (2011), Chiavenato (2009), Hay Group (2012), entre outros. A pesquisa foi descritiva, campo, desenho não experimental, transversal, questionário dirigido a 35 supervisores, com 30 itens, com escala de likert. A confiabilidade de 0,84. Como resultado, existem planos de recrutamento nas empresas e essas políticas incluem fontes de recrutamento, dentro e fora da organização, usando bancos de dados, além de ter políticas ou programas de retenção que permitam Bons funcionários; Além disso, são estabelecidos planos de incentivo salarial, promovendo o crescimento profissional do supervisor. Em termos de habilidades, trabalho em equipe, cooperação e liderança são evidentes. Concluiu-se que, nas organizações em estudo, estão orientadas a executar processos de gestão de talentos humanos de forma coordenada, em virtude da promoção do desenvolvimento de competências.
\end{abstract}

Palavras-chave: Gestão; talentos humanos; políticas; competências; gestão. 


\section{INTRODUCCIÓN:}

En la actualidad el marco de actuación de las organizaciones está definido por un entorno globalizado, ampliamente competitivo, por lo cual resulta necesario el establecimiento de estrategias para asegurar esa competitividad a través de la excelencia en el servicio, la producción y venta de productos de alta calidad, la disponibilidad del producto en el momento preciso que se genera de la necesidad del cliente, así como de los lineamientos de las empresas.

Para mantener la competitividad es necesario destacar el papel que juega el recurso humano dentro de las organizaciones, desde la cúspide de la estructura hasta los niveles inferiores del organigrama, guardadas las proporciones en cuanto a responsabilidades. En este sentido, dadas las necesidades de competitividad, las organizaciones requieren contar con los mejores elementos en cada área de trabajo que les garanticen cierta continuidad, pero en la práctica no siempre sucede así.

Es así como los trabajadores mejor preparados, por lo general buscan otras opciones que les proporcionan mayores elementos de desarrollo profesional y económico, por ende las empresas que no "sean capaces de gestionar los recursos humanos eficazmente, les resultará difícil atraer y retener a los mejores empleados, asimismo estarán orientadas al fracaso a la hora de maximizar el valor del capital humano.

Cabe destacar que existe hoy en día una guerra por talento centrado en competencia y compromiso, en este aspecto la competencia tiene que ver con las empresas que tienen el conocimiento correcto, destreza, actitud, a su vez motivan a los empleados para hacer frente al trabajo diario y futuro. Por ende el compromiso para Koontz y Weihrich (2015) viene de los empleados al sentirse respetados y tratados con dignidad. Es allí donde Recursos Humanos tiene un papel fundamental en el juego del capital intelectual. Las empresas que pueden crear políticas que incrementan la competencia y compromisos ganarán la guerra de los talentos.

A este respecto, Chiavenato (2009) señala que las políticas son reglas que se establecen para dirigir funciones y asegurar que estas se desempeñen de acuerdo con los objetivos deseados. En el caso de las relacionadas con la gestión del talento humano, constituyen una orientación administrativa para logar que empleados desempeñen funciones, orientadas a alcanzar el éxito de las funciones o tareas específicas asignadas según las exigencias del puesto de trabajo

Bajo esta premisa, Rubio y García (2014) señalan que la gestión del capital humano debe considerar dentro de sus funciones la formación y desarrollo del personal por competencias, a través de la implementación de políticas y prácticas en las cuales se fundamenten los procesos de planeación, organización, dirección y control del talento humano, con la finalidad de desarrollar determinadas características en los individuos (habilidades, destrezas, valores, hábitos, motivos), que hacen que su comportamiento sea especialmente satisfactorio en el entorno empresarial, permitiendo así el logro de los objetivos establecidos.

Esta realidad obliga al establecimiento de planes que permitan la permanencia de los talentos en la organización, dándole valor agregado a la experiencia y al conocimiento adquirido, de allí que es necesario gestionar el talento en las organizaciones para garantizar la competitividad, lo que implica el establecimiento de programas específicos de identificación, atracción y retención de talentos. Asimismo, el enfoque de competencias llegó desde la vertiente de la educación a jugar un papel fundamental en la modernización y actualización de la oferta de formación y capacitación para el trabajo. Esto valida la apreciación de Estrada y Saumett (2014), quienes en su estudio plantean que en las pequeñas unidades productivas es requerido competencias académicas, derivadas de la formación, en los directivos de este tipo de organizaciones.

En este orden de ideas, según Alles (2011) aplicar las competencias en la gestión de talento humano (en lo sucesivo GTH), se ha convertido en una buena fórmula para alcanzar un mejor aprovechamiento de las capacidades de la gente. Incorporar las competencias implica cuestionarse no solo por los resultados que se espera alcanzar, sino por la forma en que las diferentes funciones de la gente que trabaja en la empresa pueden coadyuvar a lograr tales resultados.

A criterio de Benavides (2003) dentro de estas competencias en la gestión del talento humano, destacan las competencias gerenciales, las cuales representan aquel conjunto de atributos personales de los directivos que son evidenciables, en su mandato y que le aportan al proceso, también se consideran aquellos comportamientos para lograr un desempeño eficiente. Por lo tanto, entre estas competencias laborales se incluyen elementos propios del ser, a saber, conocimientos; habilidades y destrezas: autoconcepto, rasgos y temperamento, motivos $y$ necesidades (Benavides 2003)

Con este propósito, los modelos de competencia se han fijado no solo en las competencias más evidentes que residen en las habilidades y en los conocimientos, sino que también han incluido la consideración de otras más suaves asociadas con el comportamiento y las conductas. Para Duran, Crissien, García y Virviesca (2017) el desarrollar un estilo de GTH que identifique las competencias gerenciales 
necesarias para el cumplimiento de los objetivos de la empresa y facilite el desarrollo de su gente orientado hacia esas competencias, es aplicar una GTH por competencias. Además García, Duran, Parra y Márceles (2017) manifiestan que las experiencias exitosas de los departamentos de Recursos Humanos, suelen residir en la habilidad de la organización para establecer un marco de competencias que refleje su filosofía, valores y objetivos estratégicos.

Este marco se convierte en el referente para las diferentes acciones en el ciclo de trabajo de la GTH. Es así, como los modelos actuales de GTH incluyen una función anticipativa o predictiva, una función de flujo y una de salida. Esta actividad permite planificar cuidadosamente la formación de modo que cumpla dos propósitos. Dado esto, Parra y Duran (2014) consideraron que es necesario hacer el nexo entre las competencias requeridas y las competencias disponibles, ello abre las posibilidades de desarrollar las organizaciones.

En virtud de ello se ha originado un nuevo enfoque de estudio centrado en los procesos, ello producto de la globalización, haciendo énfasis en la creación de metodologías de trabajo que apoyen los esfuerzos de ellas para cambiar, hacerse globales, flexibles, productivas, además generar estrategias orientadas al cliente. Tal y como lo plantean Prieto, Burgos, García y Rincón (2016) cuando afirman que la efectividad, productividad y competitividad son variables fundamentales del mercado nacional e internacional, razón por la cual se hace necesaria la aplicación de métodos o técnicas que permitan optimizar el desenvolvimiento de los procesos de producción, así como de administración de las organizaciones, en virtud de mejorar las actividades laborales y los procesos productivos.

Bajo este contexto, para la ejecución de los procesos organizacionales, se requiere de un talento humano comprometido e identificado con la cultura de la empresa, lo que según la convicción de Duran (2010) se destaca el rol que juega el individuo dentro de toda organización, este se perfila como el promotor del funcionamiento de las actividades inherentes a la razón de ser de la organización.

En tal sentido es de resaltar que la exigencia por parte del usuario hace necesaria la orientación y restructuración de las organizaciones hacia la excelencia de los procesos de trabajo, lo cual implica la formación capaz y comprometida con el éxito de la organización en cada área que la conforma, desde el nivel gerencial hasta el operativo.

Aunado a ello, Prieto, Villasmil y Urdaneta (2011) manifestaron que las organizaciones se han orientado a buscar nuevas alternativas de gestión para responder de manera efectiva a las variables del entorno, pero tomando como base fundamentalmente al talento humano, quien tiene a su disposición las técnicas y herramientas necesarias para ejecutar los procesos.

Es importante resaltar que para Chiavenato (2009) tener personas no significa necesariamente tener talentos, un talento es siempre un tipo especial de persona, pero no siempre toda persona es un talento, para ser talento la persona debe poseer algún diferencial competitivo que lo valores. Dentro de este marco, el talento humano es la capacidad para aprender, este pertenece principalmente a las personas puesto que el conocimiento reside en ellas.

Por otra parte, las organizaciones exitosas son particularmente aptas para reunir a distintos tipos de personas para que alcancen un objetivo común, lo cual es la esencia de la Administración de Recursos Humanos (ARH). Por lo cual la Gestión de talento Humano representa la acción y visión de las empresas, frente a su masa laboral, la cual debe ser ejecutada estratégicamente para lograr un desempeño eficiente de los trabajadores.

Una de las finalidades de la gestión humana es proyectar y desarrollar el recurso humano mediante planes tendientes a mejorar en el personal, habilidades, destrezas y competencias, con la finalidad de alcanzar un máximo rendimiento laboral. En concordancia con lo antes mencionado el empleado debe ser considerado como una inversión, ella a través del tiempo va a generar rentabilidad, no como un gasto en el que incurren las organizaciones; ella forjará un valor agregado que permitirá alcanzar objetivos.

Para ello, se requiere conocer las competencias que deben poseer los trabajadores en cuanto a las habilidades, destrezas, características, actitudes y conocimientos necesarios para el pleno desempeño de sus funciones. En base a lo planteado, se retoma el término competencia que según Hay Group (2013), son "la representación del deseo de causar impacto en los demás, o la capacidad de afectar a otra persona mediante estrategias de persuasión e influencia". Ahora bien, existen diversas y variadas definiciones en torno a las competencias laborales, las cuales se entienden como aquellas capacidades del individuo, necesarias para llevar a cabo exitosamente una tarea 0 actividad laboral plenamente identificada.

Dentro de este marco, la Competencia Laboral es una capacidad real demostrada, más que una probabilidad de éxito en la ejecución de un trabajo; debido a que el mismo concepto, en su esencia más estricta encierra diversos aspectos tales como comportamientos, facultad de análisis, toma de decisiones, transmisión de información, entre otros, considerados necesarios para el pleno desempeño de la ocupación. 
En tal sentido se deben ejecuta un proceso de direccionamiento estratégico de talento humano, tal y como lo presentan García et al (2017) donde se concibe al trabajador como el potencial a través del cual se logra la eficiencia y eficacia de la organización, proporcionándoles los métodos para optimizar su desarrollo, ello permitirá aprovechar de sus capacidades, por lo tanto de sus potencialidades.

Se destaca que el modelo de competencias, contiene varias etapas que lleva a las empresas e instituciones que lo adoptan a conocer su funcionamiento interior para verse reflejado en el mundo empresarial. Ello permite que se detecten, adquieran, potencien y desarrollen las competencias que dan valor agregado a la organización, las cuales diferencien en su sector, proponiendo un estilo de dirección donde lo primordial sea el factor humano, en el que cada persona debe aportar sus mejores cualidades a la organización.

Bajo las perspectivas de realizar sus actividades y procesos de recursos humanos fundamentado en lineamientos metodológicos gerenciales, se encuentran las empresas metalmecánica de Barranquilla, en la actualidad no cuentan con las herramientas para el cumplimiento del trabajo, debido a la falta de descripción de cargo, de puestos de trabajo, por ende los trabajadores del área no están orientado por una filosofía de gestión establecida.

En este ámbito, se ha podido observar que los profesionales que ejecutan funciones supervisorias desde el aspecto administrativo, han ido adquiriendo nuevos conocimientos, competencias y experiencias a lo largo de su carrera profesional, motivados por los requerimientos del entorno social, laboral y por la búsqueda del desarrollo personal y profesional. Asimismo, se ha podido percibir y corroborar en la dinámica diaria de los procesos ejecutados en dichas organizaciones, que el personal supervisorio que ahí labora está en una constante búsqueda de mejoras continua, desde su proceso individual, sin apoyo de la organización.

Situación que conlleva a preguntar; que pasa con la gestión del talento humano, su desarrollo, carrera, motivación y capacitación. Por ende se debe analizar de quien o quienes dependen gestionar el talento humano que cada día se convierte en algo indispensable para el éxito de las organizaciones. De acuerdo a lo planteado surge la necesidad de analizar las políticas de recursos humanos para promover las competencias gerenciales del personal supervisorio de empresas metalmecánicas en Barranquilla.

\section{MARCO TEÓRICO:}

2.1. Políticas de gestión de Talento Humano.
Aunque unas empresas son muy diferentes de otras, todas tienen una característica en común: su éxito depende, en gran medida, de su talento humano, por ello, se asume que lo más importante para el éxito de cualquier organización, son las personas que la integran. Chiavenato (2009), considera que la gestión del talento humano en las organizaciones es la función que permite la colaboración eficaz de las personas para alcanzar los objetivos organizacionales e individuales.

Es evidente que no se puede analizar de una manera aislada la gestión del talento humano, porque se encuentra englobada en el contexto general de la gestión integral de los recursos humanos, por lo tanto, esta se tiene que ver desde esa perspectiva y en cada uno de los procesos que la gestión integral del recurso humano implica. En este sentido, gestionar el talento humano se ha convertido en la clave para el éxito empresarial, es parte del reto principal que tienen que afrontar la organización.

Por lo tanto es necesario incorporar una visión más integradora en la mentalidad de los directivos y trabajadores de las diferentes organizaciones y siempre pensar que las propuestas metodológicas que se utilicen deben implementarse en su totalidad para lograr la sinergia funcional del proceso. Una vez logrado esto lo que resta es dirigir el talento hacia el destino de impacto deseado y con ello se obtendrán los resultados deseados.

Al respecto Alles (2011) define la gestión de recursos humanos como aquella acción de manejar, regir, aplicar, es decir un manejo integral del personal. Es el conjunto de políticas y prácticas necesarias para dirigir los aspectos de los cargos gerenciales relacionados con las personas, incluido reclutamiento, selección, capacitación, recompensas y evaluación de desempeño.

Además, a criterio de Dessler y Valera (2011) gestionar el talento humano se convierte cada día en algo indispensable para el éxito de las organizaciones. Tener personas no significa necesariamente tener talentos. Para ser talento, la persona debe poseer aspectos esenciales 0 algún diferencial competitivo que la valore. La dimensión que se le dé depende del enfoque estratégico y las políticas de recurso humanos que se apliquen en la organización.

Las políticas aplicadas de recursos humanos son importantes, en gran medida, que sirven para atraer, retener y motivar a los empleado, en general, se acepta que cuando se logran estos objetivos explícitos, ello afecta positivamente los resultados de la organización, a este respecto Mondy (2010) considera que las unidades que realizan las tareas de GTH han cambiado en forma trascendental en años recientes, y en la actualidad no existe un departamento típico de RH. Muchos de estos 
cambios se están realizando de manera que los profesionales de $\mathrm{RH}$ puedan desempeñar un papel más estratégico.

En este sentido, Chiavenato (2009), señala que las políticas son reglas que se establecen para dirigir funciones y asegurar que estas se desempeñen de acuerdo con los objetivos deseados. Estas constituyen una orientación administrativa para impedir que los empleados desempeñen funciones que no desean 0 pongan en peligro el éxito de las funciones 0 tareas específicas asignadas según las exigencias del puesto de trabajo.

Asimismo son consecuencia de la racionalidad, la filosofía y la cultura organizacional, lo cual en opinión de Prieto, Emonet, García y González (2015) son reglas que se establecen para dividir funciones, asegura que estas se desempeñen de acuerdo con los objetivos deseados. Constituyen orientación administrativa para impedir que los empleados desempeñen funciones que no desean 0 pongan en peligro el éxito de funciones específicas.

Bajo este contexto, las políticas son guías para la acción, sirven para dar respuestas a los integrantes 0 problemas que pueden presentarse con frecuencia, ellos obligan a que los subordinados acudan sin necesidad ante los supervisores para que estos les solucionen cada caso. Para Werther y Davis (2014) las políticas son reglas que se establecen para dividir funciones y asegurar que estos se desempeñen de acuerdo a los objetivos planteados. Además los autores proponen las siguientes políticas.

Ver Gráfico 1.

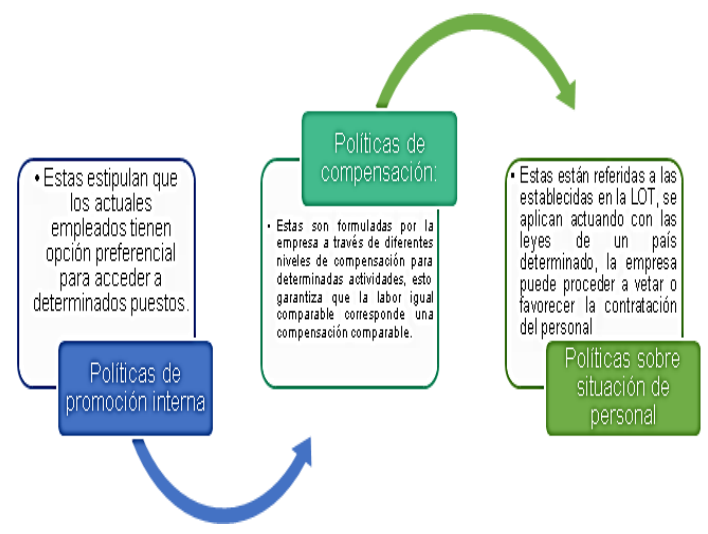

Grafico 1. Políticas de Recursos Humanos

Fuente: Elaboración propia (2017), a partir de los postulados de Brunet (2004)

Es importante que se establezcan políticas de recursos humanos para alcanzar una estructura organizacional adecuada, esto permitirá tomar decisiones rápidas sin tener que acudir a los supervisores cada vez que ocurra una situación inesperada. De acuerdo a lo planteado por Paz, Harris y García (2015) las organizaciones actuales se encuentran en un entorno de cambios permanentes, y la necesidad de supervivencia en este hace de la toma de decisiones un instrumento estratégico gestor y garante de ventajas competitivas.

Asimismo, Wherter et al (2014) sostiene que estas políticas son consecuencia de la racionalidad, la filosofía y la cultura organizacional, son reglas que se establecen para dirigir funciones y asegurar que estas se desempeñen de acuerdo con los objetivos deseados. Constituyen una orientación administrativa para impedir que los empleados, desempeñen funciones que no desean o pongan en peligro el éxito de funciones específicas. Sobre la base de la idea expuesta, cabe agregar que el autor califica las políticas de la siguiente manera, Ver gráfico 2.

Grafico 2. Políticas de gestión de Talento Humano

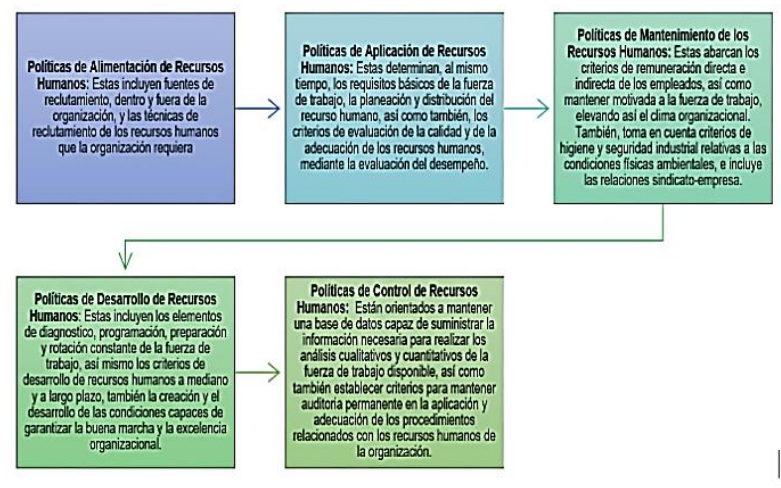

Fuente: Elaboración propia (2017) fundamentado en la teoría de Wherter y Davis (2014).

De acuerdo con lo planteado, se concluye que las políticas de recursos humanos son la manera de cómo las empresas aspiran que sus miembros alcancen los objetivos organizacionales e individuales mediante normas, reglas, códigos éticos, que van a permitir que exista una buena relación patrón-empleado, así como también la relación entre accionistas, proveedores y consumidores.

\subsection{Competencias gerenciales.}

Hoy en día, las empresas enfrentan crecientes desafíos competitivos ligados a los mayores requerimientos de calidad de servicio por parte de sus clientes y a la necesidad de mejoramiento de su gestión, procesos productivos y productos. Estas competencias dan lugar a un desempeño superior en una serie de roles empresariales. Según Chiavenato (2009), una competencia es un repertorio de comportamientos capaces de integrar, movilizar y transferir conocimientos, habilidades, juicios y 
actitudes que agregan valor económico para la organización y valor social para la persona.

Al respecto para Alles (2011) el termino competencia hace referencia a características de personalidad, devenidas comportamientos, que generan un desempeño exitoso en un puesto de trabajo. Competencias representan un rasgo de unión entre las características individuales y las cualidades requeridas para conducir muy bien las misiones profesionales prefijadas. Según Hay Group (2013) las competencias pueden consistir en motivos, rasgos de carácter, conceptos de uno mismo, actitudes 0 valores, contenido de conocimientos 0 capacidades cognoscitivas o de conducta, es decir, cualquier característica individual que se pueda medir de un modo fiable, además pueda demostrar que diferencia de una manera significativa entre los trabajadores con un desempeño excelente de los adecuados 0 entre los trabajadores eficaces e ineficaces.

De acuerdo a lo anteriormente, las competencias constituyen la base esencial para definir los perfiles técnicos y estratégicos que necesita una organización para materializar con éxito sus objetivos, una vez identificada, se usan como guía o patrón para poder seleccionar personal adecuado, del mismo modo desarrollar al personal, así como, fomentar la formación y sus habilidades pero además el entrenamiento para garantizar la continuidad operativa de la organización.

Por otra parte, el conocimiento de las personas, sus destrezas, habilidades, motivos, rasgos y el concepto de sí mismo, tal y como lo han sustentado Duran y Parra (2014), corresponden a elementos que distinguen a una persona de otra, es decir, cualquier característica individual, para medir de un modo confiable, pero también pueda demostrar la diferencia significativa entre los trabajadores que reflejan su desempeño excelentemente.

En este sentido, Beltrán. Contreras y Chipatecua (2012), manifiestan que las competencias laborales constituyen la agrupación de capacidades que posibilitan el desarrollo y adaptación de la persona al puesto de trabajo, asi como el conjunto de saberes entrelazados de forma sistémica que se exponen durante el desempeño laboral en correspondencia con el entorno, y que proporcionan calidad, eficiencia y productividad a la organización; lo que se pone de manifiesto en los conocimientos, habilidades y actitudes.

Asimismo, se destaca que las competencias gerenciales se agrupan en dos grandes categorías: las competencias hard, referidas al conjunto de conocimientos y de habilidades que posibilitan a las personas la ejecución óptima de sus trabajo y, las segundas, a las competencias soft, que engloban factores de naturaleza actitudinal que tienen validez en diferentes contextos, vislumbrándose como elementos estratégicos de las organizaciones en sus planteamientos.

Para De la Varga y Galindo (2015), la conjunción de ambas ideas, de un lado, la importancia de las competencias directivas como herramienta fundamental para garantizar la competitividad de las organizaciones y, de otro, la conceptualización de las competencias en base a conocimientos, habilidades y actitudes, sirve de base para definir un modelo de investigación. La primera variable hace referencia a los conocimientos y habilidades de los directivos, correspondientes, respectivamente, a los conocimientos que posibilitan a los directivos la ejecución óptima de su trabajo y a la utilización de dichos conocimientos mediante el despliegue de las habilidades necesarias para el desarrollo de su función.

La segunda variable hace alusión a la actitud directiva, que representa la disposición favorable del directivo hacia el desarrollo de determinados comportamientos que resultan adecuados a las normas y a la cultura de la organización. Ademas estos autores consideraron una tercera variable, correspondiente a la capacidad para innovar de la organización, considerada como la "capacidad para generar o adoptar e implementar ideas diferentes y mejores de manera sostenible" sustentados en (Hurley y Hult, 1998; Lawson y Samson, 2001).

\section{Grafico 3. Competencias gerenciales}

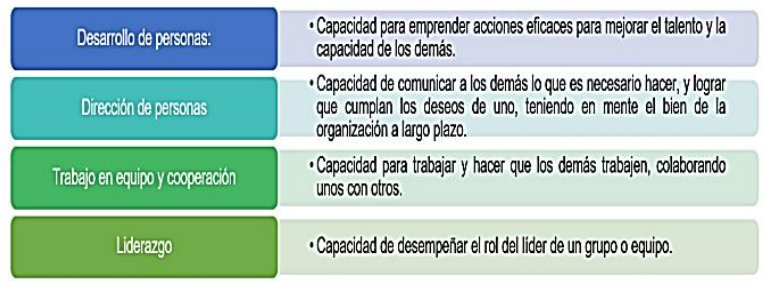

Fuente: Elaboración Propia (2017). Sustentado en Benavides y Quintana (2003).

Bajo este contexto, las competencias gerenciales, representan el conjunto de atributos personales evidenciables, que se aportan al trabajo, también se consideran los comportamientos para lograr un desempeño eficiente. Por lo tanto las competencias laborales incluyen elementos propios del ser, a saber, conocimientos; habilidades y destrezas: autoconcepto, rasgos y temperamento, motivos y necesidades (Benavides 2003). Por su parte conceptualiza las competencias directivas como comportamientos observables y habituales que posibilitan el éxito de una persona en su función directiva. Añade que estas competencias son más genéricas. En este sentido, la función directiva consiste en diseñar estrategias que produzcan valor económico, desarrollando las capacidades de sus empleados y uniéndolos con la 
misión de la empresa. Cardona (2008) incluye junto con la función directiva otra denominada intrategica.

Siguiendo con lo expuesto, la dimensión intratégica se dirige al desarrollo de los empleados y al incremento de su grado de compromiso con la misión de la empresa. El mismo autor deduce de este análisis dos tipos de competencias directivas: la intrategica, la responsable de generar el grado de confianza y compromiso para con la empresa y la estratégica la que obtiene buenos resultados económicos. A estas agrega la competencia de eficacia personal, la cual define como aquellos hábitos que facilitan una relación eficaz de la persona con su entorno. Esta mide la capacidad de autodirección, según expresa Cardona (2008), capacidad imprescindible para dirigir a otras personas.

Por consiguiente, competencias estratégicas es el grupo de competencias directivas referidas a la capacidad estratégica de un directivo y su relación con el entorno de la empresa. En este orden de ideas, las empresas metalmecánica han experimentado sustanciales cambios, originados por la competitividad exigida en los mercados globales, así como el rápido avance de la tecnología. Se requieren nuevas organizaciones basadas en redes y equipos de trabajo, que usen tecnologías, procesos flexibles, los cuales deben tener en cuenta a los clientes.

Por ende, en las organizaciones se requiere personas versátiles y polivalentes, que sepan identificar oportunidades para crear servicios, asociarse con otros 0 generar unidades beneficiosas de carácter asociativo y cooperativo. Personas que sean capaces de adaptarse a los cambios del entorno, de auto dirigirse y autoevaluarse, de relacionarse apropiadamente con otros y de aprender cada vez más sobre su trabajo.

Para vincular personal adecuado a la empresa, este departamento empleador, tomara en cuenta, de manera especial, las condiciones personales que involucran competencias clave, como el comportamiento ético, las capacidades para comunicarse, trabajar en equipo y manejar recursos e información, ello redundara en aspecto positivo y mayor productividad.

\section{METODOLOGÍA:}

La investigación se califica como una investigación de tipo descriptiva, la misma se orientó a analizar las políticas de gestión de talento humano en el desarrollo de las competencias gerenciales del personal supervisorio en empresas metalmecánica en Barranquilla Colombia. Asimismo es una investigación de Campo, con un diseño no experimental, transversal, la recolección de datos se realizó en un único momento, obteniendo de esta forma una sola medición.

En cuanto a la población, se consideraron 35 supervisores de empresas. Siendo las unidades informantes, los gerentes, propietarios, administradores 0 los encargados de las empresas. En cuanto a la recolección de datos, se utilizaron dos cuestionarios uno para analizar las políticas de gestión de TH y otro para determinar las competencias. La confiabilidad del instrumento se determinó con el método Alfa Crombach, arrojando como resultado rtt: 084, lo que determina que el instrumento es altanamente confiable. Los resultados se presentaron con estadísticas descriptivas y medidas de tendencia central, además se presenta la discusión de los datos.

\section{RESULTADOS Y DISCUSION:}

Tabla 1. Políticas de gestión de talento humano

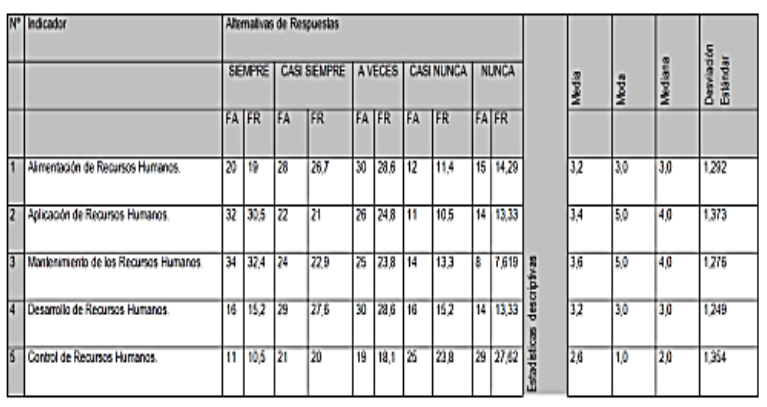

\section{Fuente: Elaboración Propia (2017)}

En la tabla 1 se muestran los resultados correspondientes a la dimensión Políticas de gestión de talento humano, la misma está compuesta por cinco indicadores:

-Alimentación de recursos humanos, en este ámbito se genera si existe un plan de captación de candidatos a fin de tener disponibilidad al momento de presentarse un puesto vacante, si dan a conocer el plan de captación de posibles candidatos para cargos vacantes, 0 si la organización realiza durante la captación de personal avisos de prensa, revisión de base de datos. En este sentido los resultados fueron que un $28,6 \%$ considero que a veces se lleva a cabo esta situación, un $26,7 \%$ considero la opción casi siempre, un $19,05 \%$ se inclina por la opción siempre, además un $14,29 \%$ y $11,43 \%$ por las categorías nunca y casi nunca. Logro un media de 3,2, el valor moda y mediana se situaron en 3 , y la desviación estándar en 1,29 .

Al respecto, la mayoría de los encuestados considera que si se ejecutan esas opciones, por ende se cita Chiavenato, (2009) estas políticas incluyen fuentes de reclutamiento, dentro y fuera de la organización, y las 
técnicas de reclutamiento de los recursos humanos que la organización requiera.

-Aplicación de Recursos Humanos. Para este indicador se obtuvo como resultado que un $30,47 \%$ de los encuestados asumió que siempre esta se ejecuta, mientras que un $24,76 \%$, consideran que a veces este paso se realiza, asimismo $21 \%, 13,33 \%$ y $10,47 \%$ por las opciones casi siempre, nunca y casi nunca, respectivamente, al ser interrogados sobre el hecho de que para retener a un empleado la organización debe ofrecerle las condiciones ambientales y psicológicas satisfactorias.

Por otra parte para las actividades a desarrollar en su puesto de trabajo, además si cuentan con políticas 0 programas de retención que permita mantener a los buenos empleados, aunado a la conveniencia para la institución de conocer las opciones disponibles en el entorno laboral externo que pueda utilizar en pro de la labor que lleva a cabo. Asimismo presento una mediana 3,4 , una moda de 5 , una mediana de 4 , una desviación estándar de 1, 37 .

Se ejecuta constantemente esta política, dado que para retener a un empleado la organización le ofrece las condiciones ambientales y psicológicas satisfactorias, además cuentan con políticas o programas de retención que permita mantener a los buenos empleados, dan a conocer las opciones disponibles en el entorno laboral externo que pueda utilizar en pro de la labor que lleva a cabo.

Esta información se sustenta en los aspectos teóricos de Wherter y Davis (2014), quien sostiene que dichas políticas determinan los requisitos básicos de la fuerza de trabajo, la planeación y distribución del recurso humano, así como también, los criterios de evaluación de la calidad y de la adecuación de los recursos humanos, mediante la evaluación del desempeño.

- Mantenimiento de los Recursos Humanos, a este respecto se involucraron los siguientes aspectos; realización de estudios de mercado para establecer el salario del personal administrativo supervisorio, se establecen planes de incentivos salariales, la empresa tiene establecida una relación equitativa entre el sueldo que devenga usted y la función que ejecuta. Los resultados se inclinaron a un $32,38 \%$ de encuestados pronunciados por la opción siempre, el $23,81 \%$.

Asimismo por la opción a veces, un 22,9\%, 13,33\% y $7,61 \%$ por las categorías casi siempre, casi nunca y nunca respectivamente. Además se obtuvo una media de 3,6, una moda de 5 , una mediana de 4 y una desviación estándar de 1,27.
A este respecto se sugirió la realización de estudios de mercado para establecer el salario del personal administrativo supervisorio, así como al establecimiento de planes de incentivos salariales, en función de una relación equitativa entre el sueldo devengado y función ejecutada. Los resultados al hecho de ser una política muy tomada en cuenta. Tal y como lo plantea Wherter y Davis (2014), estas abarcan los criterios de remuneración directa e indirecta de los empleados, así como mantener motivada a la fuerza de trabajo, elevando así el clima organizacional. También, toma en cuenta criterios de higiene y seguridad industrial relativas a las condiciones físicas ambientales, e incluye las relaciones sindicato-empresa.

- Desarrollo de Recursos Humanos, en este indicador los encuestados se inclinaron en un $28,57 \%$ por la opción a veces, un $27,6 \%$ considero la opción casi siempre, un $15,23 \%$ manifestó que siempre se ejecuta, mientras que el otro $15,23, \%$ asumió que casi nunca, pero además un $13,33 \%$ seleccionó la opción nunca.

Con respecto la promoción del crecimiento profesional del supervisor, la conveniencia de conocer las opciones disponibles en el entorno laboral externo que pueda utilizar en pro de la labor que lleva a cabo, así como si los objetivos de la empresa van en pro de la consecución de la misión que persigue la misma, aunado a ello este indicador presento una media de 3,2, un valor moda y mediana de 3 y una desviación estándar de 1,24.

Los encuestados se inclinaron hacia la opción siempre, referido a la promoción del crecimiento profesional del empleado, conocer las opciones disponibles en el entorno laboral externo, así como si la alineación de los objetivos de la institución con la misión, en este sentido Wherter y Davis (2014), estas políticas incluyen los elementos de diagnóstico, programación, preparación y rotación constante de la fuerza de trabajo, así mismo los criterios de desarrollo de recursos humanos a mediano y a largo plazo, también la creación y el desarrollo de las condiciones capaces de garantizar la buena marcha y la excelencia organizacional.

- Control de Recursos Humanos; a este respecto los datos arrojados determinaron que un $27,61 \%$ de los encuestados considero que nunca se cumple este indicador, referido a las estrategias de gestión utilizadas por la institución favorezcan lo propósitos de la organización, además si hacerle seguimiento a las actividades que desarrolla el personal supervisorio le permite mantener ventaja competitiva sobre los procesos, aunado al hecho que para gestionar el talento humano es importante el uso de políticas disciplinarias. Mientras que el $23,8 \%$ considero casi nunca, el $20 \%$, casi siempre aunado al $18,09 \%$ y $10,47 \%$ se inclinan por las opciones, a veces y siempre respectivamente. Así mismo, presenta una 
media de 2,6, una moda de 1, la mediana es de 2 y una desviación estándar de 1,35.

Los datos arrojados determinaron que la mayoría de la población considero que no se cumplen con esas políticas, dado que las estrategias de gestión utilizadas no favorecen lo propósitos de la organización, no le hacen seguimiento a las actividades que desarrolla el personal supervisorio, además que las políticas disciplinarias no están bien planteadas. Resultado que se contradice con los postulados de Wherter y Davis (2014), quien sustenta que esta política busca mantener una base de datos capaz de suministrar la información necesaria para realizar los análisis cualitativos y cuantitativos de la fuerza de trabajo disponible, así como también establecer criterios para mantener auditoria permanente en la aplicación y adecuación de los procedimientos relacionados con los recursos humanos de la organización.

TABLA 2. Competencias gerenciales

\begin{tabular}{|c|c|c|c|c|c|c|c|c|c|c|c|c|c|c|c|}
\hline \multirow{3}{*}{$\mathrm{N}^{0} \mid \mathrm{V}$} & \multirow{3}{*}{ Indcador } & \multicolumn{10}{|c|}{ Alternativas de Respuestas } & \multirow{3}{*}{8} & & \multirow{3}{*}{$\sum_{0}^{\infty}$} & \multirow{3}{*}{ 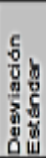 } \\
\hline & & \multicolumn{2}{|c|}{ Siempre } & \multicolumn{2}{|c|}{ Casi siempre } & \multicolumn{2}{|c|}{ Aveces } & \multicolumn{2}{|c|}{ Casinunce } & \multicolumn{2}{|c|}{ Nunca } & & & & \\
\hline & & FA & FR & $\mathrm{FA}$ & FR & FA & FR & FA & FR & $\mathrm{FA}$ & FR & & & & \\
\hline 1 & $\begin{array}{l}\text { Deserrollo } \\
\text { personass }\end{array}$ & 58 & 55 & 28 & 27 & 14 & 133 & 5 & 47 & 0 & 0 & 43 & 5 & 5 & 09 \\
\hline 2 & $\begin{array}{l}\text { Dirección } \\
\text { personass }\end{array}$ & 30 & 31 & 31 & 20,5 & 21 & 20 & 16 & 15,2 & 2 & 1,9 & 3,8 & 5 & 4 & 1,1 \\
\hline 3 & $\begin{array}{l}\text { Trabaio en equipo y } \\
\text { cosopersición }\end{array}$ & 43 & 41 & 20 & 19 & 15 & 14,2 & 25 & 24 & 3 & $2, B$ & 3,7 & 5 & 4 & 1,3 \\
\hline 4 & Lixlerargo & 的 & 64,7 & 26 & 24,7 & 8 & 7,6 & 5 & 4.7 & 0 & 0 & 4,5 & 5 & 5 & 0,8 \\
\hline
\end{tabular}

Fuente: Elaboración Propia (2017)

Para sustentar la variable competencia se despliega el objetivo orientado a identificar las competencias gerenciales del personal supervisorio de empresas metalmecánica, iniciando con los elementos 0 competencias identificadas

Desarrollo de personas. En este aspecto la población orientó sus respuestas de la siguiente manera, un 55\% consideró que siempre se promueve el desarrollo de las personas, un $27 \%$ casi siempre, un $14 \%$ a veces y el $4,7 \%$ restante manifestó que casi nunca se cumple; a este indicador se le vincula con los siguientes aspectos; capacidad de integrar las actividades con los objetivos, promover la iniciativa del empleado para el desarrollo del proceso.

Asimismo si el talento humano genera aportes en el desarrollo de los procesos laborales. Aunado a ello, las medidas de tendencias resultaron de la siguiente manera, la media fue de 4,3 , la moda y mediana obtuvo un valor de
5 , en tanto la desviación estándar fue de 0,9 , siendo un valor muy bajo con respecto al promedio.

-Dirección de personas. Para este indicador el $31 \%$ de los encuestados consideraron la opción siempre, un 29,5\% casi siempre, $20 \%$ a veces, el 15,2 seleccionó la alternativa casi nunca, mientras que solo el 1,9\% manifestó por la opción nunca, en referencia a los ítems relacionados la utilización del conocimiento para agregar valor a las tareas desempeñadas, además el desarrollo del conocimiento del empleado es asumido como una inversión.

En el mismo orden de ideas, desarrollar habilidades en el empleado, como inversión para la empresa. En este sentido, las tendencias se orientan a la existencia de esta competencia gerencial. Por otra parte los resultados asociados a las medidas de tendencia central y de dispersión, los resultados fueron para la media 3,8, para la moda, 5, el valor de la mediana fue 4, asimismo la desviación estándar fue 1,1.

Trabajo en equipo y cooperación, asociado a este Idicador estuvieron los ítems siguientes: la integración de 1 comunicación permite el aprendizaje del equipo, la ansferencia de conocimiento al equipo permite el logro de letas organizacionales, promoción de la interacción del ælento humano para compartir las ideas.

A ello los encuestados respondieron de la siguiente ranera, el $41 \%$ considero que siempre lo hacen, un $24 \%$ lanifestó que casi nunca; pero además un $19 \%$ seleccionó 1 alternativa casi siempre, mientras un 14,2 y 2,8 consideraron las opciones a veces y nunca respectivamente. Con respecto a los valores de medida el medio arrojo un valor de 3,7, la moda 5 , asimismo la mediana se ubicó en 4, finalmente para este indicador la desviación estándar mostro un valor de 1,3.

-Liderazgo. Este indicador estuvo relacionado con los siguientes aspectos, colaboración del supervisor con otros en pro de ser más competitivo, fijación de objetivos, orientación y seguimiento de las acciones, del personal a su cargo, para liderizar al equipo de trabajo, a ello contestaron en un $64,7 \%$ y $24,7 \%$ siempre y casi siempre, por otra parte el $7,6 \%$, así como el $4,7 \%$ a veces y casi nunca. Los resultados de las medidas estadísticas fueron una media de 4,5 , la moda y mediana 5 , por último la desviación estándar resulto 0,8

Competencias gerenciales de la población objeto de estudio, incluyendo elementos claves para la gerencia de hoy, entre ellas está el desarrollo de personas, asociado a la capacidad de integrar las actividades con los objetivos, promover la iniciativa del empleado para el desarrollo del proceso. 
Los resultados generaron aspectos de prevalencia y ocurrencia continua, dado que la mayoría considero que siempre se aplica esta competencia. Dentro de este marco, Benavides y Quintana (2003) manifiesta que los supervisores deben tener la capacidad para emprender acciones eficaces para mejorar el talento y la capacidad de los demás.

Por otra parte se analiza la dirección de personas, el resultado indica que este aspecto prevalece y se aplica continuamente, la mayoría de los encuestados consideraron la opción siempre y casi siempre, referido a la utilización del conocimiento para agregar valor a las tareas desempeñadas, el desarrollo del conocimiento del empleado es asumido como una inversión, sumado a desarrollar habilidades en el empleado, como inversión para la institución. En este sentido, las tendencias se orientan a la existencia de esta competencia gerencial. Para Benavides y Quintana (2003), la dirección de personas debe orientarse a la capacidad de comunicar a los demás lo que es necesario hacer, y lograr que cumplan los deseos de uno, teniendo en mente el bien de la organización a largo plazo.

En el mismo orden de ideas, la competencia gerencial trabajo en equipo y cooperación, se asoció a la integración de la comunicación para mejorar el aprendizaje del equipo, la transferencia de conocimiento al equipo permite el logro de metas organizacionales, y la promoción de la interacción del talento humano para compartir las ideas, al respecto los encuestados respondieron la opción siempre, es decir que existe el trabajo en equipo y la cooperación. Benavides y Quintana (2003), sugiere que la competencia de un gerente se orienta hacia el trabajo en equipo y cooperación, generando la capacidad para trabajar y hacer que los demás trabajen, colaborando unos con otros.

Finalmente el indicador liderazgo, el cual se relacionó con la colaboración del supervisor con otros en pro de ser más competitivo, la fijación de objetivos, orientación y seguimiento de las acciones, del personal a su cargo, para liderizar al equipo de trabajo, a ello contestaron la mayoría en un $64,7 \%$ y $24,7 \%$ siempre y casi siempre, dejando muy claro que una de las competencias gerenciales que prevalecen es el liderazgo.

Aunado a ello, Benavides y Quintana (2003), manifiesta que el liderazgo, es la capacidad de desempeñar el rol del líder de un grupo o equipo. Además las competencias laborales o gerenciales, representan el conjunto de atributos personales evidenciables, que se aportan al trabajo, también se consideran los comportamientos para lograr un desempeño eficiente.

\section{CONCLUSIONES:}

Se determinaron las políticas de gestión de talento humano, generándose que se plantean y ejecutan la alimentación de recursos humanos asociados a un plan de captación de candidatos y que se ejecuta la captación de personal a través avisos de prensa, revisión de base de datos. En cuanto a la aplicación de Recursos Humanos, esta política se aplica en forma continua dado que los institutos buscan ofrecer las condiciones ambientales y psicológicas adecuadas al personal, aunado a programas de retención a los buenos empleados.

En el marco de la política de mantenimiento de los Recursos Humanos, se busca establecer el salario del personal administrativo supervisorio, así como al establecimiento de planes de incentivos salariales, tratando de tener una relación equitativa entre el sueldo devengado y función ejecutada. Con respecto al desarrollo de Recursos Humanos, existe la promoción del crecimiento profesional, dando a conocer las opciones disponibles en el entorno laboral externo para que puedan capacitarse, de esta manera puedan alinearse con los objetivos de la institución.

En referencia al control de Recursos Humanos; en forma general no se cumplen, puesto que las estrategias de gestión utilizadas no son las más acordes para el cumplimiento de los propósitos de la organización, por otro lado no le hace seguimiento a las actividades que desarrolla el personal supervisorio, aunado al hecho de que las políticas disciplinarias no están bien planteadas.

Asimismo, respecto a las competencias gerenciales del personal supervisorio, quienes están orientados hacia el trabajo en equipo y cooperación, generando la capacidad para trabajar y hacer que los demás trabajen, colaborando unos con otros, promoviendo al líder, en fin se concluye que estas competencias representan el conjunto de atributos personales evidenciables, que se aportan al trabajo, también se consideran los comportamientos para lograr un desempeño eficiente.

\section{REFERENCIAS:}

Alles, Martha (2011). Dirección Estratégica de Recursos Humanos. Gestión Por Competencias. Buenos Aires. Nueva Edición. Editorial Granica.

Alles, Martha (2011). Desarrollo del Talento Humano, Basado en Competencias. Buenos Aires. Nueva Edición. Editorial Granica.

Benavides C y Quintana C, (2003) Gestión del conocimiento y Calidad total, ediciones Díaz de santos, Madrid, España 
Cardona P. (2008) Paradigmas del liderazgo. Claves de la dirección de personas. McGraw - Hill Madrid.

Chiavenato, Idalberto (2009). Gestión del Talento Humano. México. Tercera Edición. Editorial Mc Graw Hill.

Chiavenato, A. (2009). Administración de Recursos Humanos. México: Editorial Mac Graw Hill Hispanoamericana S.A.

De la Varga Salto José María y Galindo Reyes Fuensanta C. (2015). Competencias soft sobre competencias hard: análisis de la capacidad para innovar en los chefs de la alta cocina española. Innovación y gestión del conocimiento. Departamento de Economía y Administración de Empresas Facultad de Comercio y Gestión. Universidad de Málaga

https://riuma.uma.es/xmlui/bitstream/handle/10630/9339/IN NOVACI $\%$ C $3 \% 93$ N\%20EN\%20LA $\% 20$ ALTA $\% 20$ COCINA\%20ESPA\%C3\%910LA\%20HISPANOLUSAS.pdf?sequence=

Dessler, G. y Valera R. (2011). Administración de Recursos Humanos. México: Editorial Pearson. 137p.

Durán, S. (2010). Diversidad Cultural y Socialización Organizacional del personal administrativo de las universidades privadas del municipio Maracaibo. Trabajo especial de grado para optar al título de Dr. En ciencias gerenciales. Venezuela: Universidad Dr. Rafael Belloso Chacín., 245.

Durán, S. \& Parra, M. (2014). Diversidad Cultural para promover el desarrollo de habilidades sociales en educación superior. Cultura Educación y Sociedad, 5(1), 55-67. 2014. Recuperado Enero de 2017.

http://revistascientificas.cuc.edu.co/index.php/culturaeduca cionysociedad/article/view/995.

Duran, S.; Crissien, J., Virviesca, J. y García, J. (2017). Estrategias gerenciales para la formación de equipos de trabajo en empresas constructoras del Caribe Colombiano. Revista Espacios Volumen 38 numero 13. Página 26

Duran S, García J \& Parra Margel. (2017) Gerencia social y motivación laboral en organizaciones de salud pública. p 38-64. En: Desarrollo organizacional y gestión humana en contextos globalizados.

Compiladores: Ronald Prieto Pulido Ana María Cazallo Antúnez. Editorial universidad Simón Bolívar, Barranquilla Colombia.

Estrada López, H. y Saumett España, H. (2014). Direccionamiento estratégico en pequeñas unidades productivas del sector lácteo del departamento del Atlántico, Colombia, en Desarrollo Gerencial Revista de la Facultad de Ciencias Económicas de la Universidad Simón Bolívar 6 (2), 16-40.

García, J., Duran, S., Parra, M. y Márceles, V. (2017) Dirección estratégica del talento humano para el fomento de valores en los cuerpos policiales, Revista Espacios Numero 38 (32) paginas 16-28

González, O. V., \& Montejo, M. L. Z. (2017). La gestión de capital humano por competencias laborales de los profesores universitarios con un enfoque estratégico. Revista Cubana de Contabilidad y Finanzas. COFIN HABANA, (1), 3545.http://ojs.uh.cu/CofinHabana/index.php/RCCF/ article/view/207/203

Hay Group (2013). Las competencias: Clave para una Gestión Integrada de los Recursos Humanos. España: Editorial Deusto.

Koontz, H.; Weihrich, H. y Cannice, M. (2015). Administración. Una perspectiva global. España. Mc Graw Hill

Mondy Wayne (2010). Administración de Recursos Humanos. Editorial Pearson Hall, México.

Parra, M., \& Durán, S. (2014). Desarrollo organizacional y estrategias de tecnoformación en instituciones de educación superior colombianas. Dictamen Libre, 14(14/15), 14-23. Recuperado 11 de marzo de 2017. En:

http://www.unilibrebaq.edu.co/ojsinvestigacion/index.php/di ctamenlibre/article/view/518

Paz Annherys, Harris Jaiham \& García, J. (2015). Toma de decisiones: reto para crear ventajas competitivas en las distribuidoras de alimentos gourmet. Desarrollo Gerencial, 7(2).

Paz, A. A., Núñez, M. G., \& García, J. (2016). Rol del liderazgo ético en organizaciones académicas. Opción, 32(12). Universidad del Zulia, Maracaibo Venezuela.

Prieto Pulido, R. A., Villasmil, M., \& Urdaneta, L. (2011). Gestión humana en organizaciones postmodernas. Base fundamental hacia la excelencia organizacional. CICAG, 8(2), 1-12.

Prieto, R., Emonet, P., García, J., \& González, D. (2015). Cambio organizacional como estrategia de gestión en las empresas mixtas del sector petrolero. Revista de Ciencias Sociales, 21(3). Recuperado 10 de marzo de 2017 en: http://200.74.222.178/index.php/rcs/article/view/2 0258 
ISSN: 1794-9920 Enero - Abril de 2017

Volumen 17 Número 2, Año 2017 Págs. 130-141

Prieto, R., Burgos, C., García, J., \& Rincón, Y. (2016). Mercadeo interno para optimizar la calidad de servicio en la banca universal. Revista Venezolana de Gerencia. Vol. $21 \mathrm{~N}^{\circ} 73$. Universidad del Zulia. Maracaibo-Venezuela. Pp.102-119.

Rubio, P. \& García, J. (2014). Gestión de la formación por competencias en operarios de alimentación como ventaja competitiva de comedores industriales en empresas privadas. Revista CICAG, Volumen 12, Edición 1, 207-230

Werther y Davis (2005). Administración de Recursos Humanos. México D.F.: Editorial Mac Graw Hill. 